\title{
Virgin Olive Oils: Environmental Conditions, Agronomical Factors and Processing Technology Affecting the Chemistry of Flavor Profile
}

\author{
Nicola Caporaso ${ }^{1,2}$ \\ ${ }^{1}$ University of Naples Federico II, Department of Agriculture, Via Università 100, Portici 80055 (NA), Italy \\ ${ }^{2}$ The University of Nottingham, Division of Food Sciences, Sutton Bonington, Loughborough, LE12 5RD, United Kingdom
}

'Correspondence to:

Nicola Caporaso

The University of Nottingham

Division of Food Sciences, Sutton Bonington

Loughborough, LE12 5RD, United Kingdom

E-mail: nicola.caporaso3@unina.it

Received: December 20, 2015

Accepted: February 09, 2016

Published: February 11, 2016

Citation: Caporaso N. 2016. Virgin Olive Oils: Environmental Conditions, Agronomical Factors and Processing Technology Affecting the Chemistry of Flavor Profile. J Food Chem Nanotechnol 2(1): 21-31.

Copyright: : (C) 2016 Caporaso. This is an Open Access article distributed under the terms of the Creative Commons Attribution 4.0 International License (CC-BY) (http://creativecommons. org/licenses/by/4.0/) which permits commercial use, including reproduction, adaptation, and distribution of the article provided the original author and source are credited.

Published by United Scientific Group

\section{Abstract}

Olive oil, and especially virgin olive oil (VOO) and extra VOO, has great interest worldwide because of its unique fatty acid profile, phenolic compounds with positive health properties for the human health and appreciated aroma profile.

However, the composition of VOO can change dramatically depending on the olive variety, field management, olive fruit maturity degree, harvesting and processing, which can affect its final characteristic flavour. At industrial level, the extraction conditions applied can affect the concentration and composition of phenolic and volatile compounds, with possible negative consequence and the formation of off-flavours. Olive crushing, malaxation, centrifugation, filtration and storage were reported to be all possible factors affecting VOO flavour.

The present review paper describes the factors affecting the virgin olive oils composition, with a focus on the aroma compounds, biophenols and the resulting sensory profile. A particular focus was put on the volatile and phenolic compounds and their relation with the sensory description and impact of the final flavour. In conclusion, many factors still need to be studied to fully understand the complex composition and interactions of this appreciated product. This information is also relevant and useful both for scientific knowledge and for its industrial application, as producers can modulate the final characteristics of the VOO.

\section{Keywords}

Olive oil quality, Aroma compounds, Olive oil chemistry, Olive oil phenolics, Extraction technology

\section{Introduction}

Olive oil has a limited production with respect to other vegetable oils, but it has a paramount importance in terms of economic significance in the producing countries and interest for consumers worldwide, which is driven by its health properties and peculiar sensory properties. The major olive oil production is localised in the Mediterranean area, being the European Union (EU) the first producer with almost 2.5 million tons in 2013/2014. After non-European countries, Syria, Turkey and Tunisia are the other important producing countries, with $180,000,135,000$ and 70,000 tons in 2013/2014, whereas a great year-byyear variability is observed because of the olive plant physiology. The EU accounts to $80-90 \%$ of the world olive production, and specifically Spain, Italy and Greece produce 45,25 and $20 \%$ of the world olive oil production, respectively [1].

Olive oil is the product obtained from olive fruit and it is mainly composed by triacylglycerols, which accounts to $98 \%$ of the total composition. The remaining 
fraction comprises free fatty acids, phenols, tocopherols, sterols, phospholipids, waxes, squalene and other hydrocarbons.

For "olive oil", many commercial categories exist: olive oil itself has been defined as a mixture of oil obtained from olives and that undergo refining process, with virgin olive oil (VOO). This latter is a product that is extracted from olives without any chemical process. Among VOOs, extra virgin olive oil has particularly high standards both in terms of composition, namely acidity, peroxide values and oxidation indices, as well as sensory characteristics assessed by recognised panels [2].

Several studies have reported on the quality of extra VOOs from the retail market, as this is relevant for the consumers in terms of the oil composition, health benefits and organoleptic properties [3]. However, it is important to understand the compositional changes due to other factors that influence VOO composition, including the agronomical practices and extraction technology. The olive variety, or "cultivar", the climatic conditions and geographical location of the olive orchard, as well as the agronomic practices and olive ripening degrees influence the final composition of the oil and therefore its flavour $[4,5]$. All these factors are described in the present review paper.

Virgin olive oil (VOO) contains an unsaponifiable fraction including waxes, phospholipids, phenolics, pigments and carotenoids. The most abundant ones are represented by phenolic compounds (oleuropein aglycone, hydroxytyrosol, lignani, ecc.), tocopherols, squalene and $\beta$-sitosterol [6].

The known antioxidant activity of olive oils is mainly due to the presence of phenolic and ortho-diphenolic compounds, and secondarily to the fatty acid composition [7]. Olive fruit contrary to other vegetable fats which usually derive from seeds - is properly a fruit. The major olive components are water (40-70\%) and fat (6-25\%), mainly present in the mesocarp. The fruit also contains simple sugars, organic acids, nitrogen compounds and bio phenols. Particularly important for olive composition is the phenolic fraction, mainly constituted by oleuropein, demethyl oleuropein and verbascoside. These compounds are present in concentration comprised between 0.5 and $2.5 \%$ of the fresh weight. The oil fraction is present as oil droplets in the pulp (16.5-23.5\% fresh weight), while little amounts are also found in the seed (1-1.5\%).

An eco-physiological role of the phenolic compounds in many fruits and vegetables seems to be related to their protection against insects or other plants "enemies". Indeed, the reaction of polyphenols after their esterification by esterase's during fruit drupe maturation exerts a plant defence mechanism against biotic attacks [8].

Once oleuropein and ligstroside are accumulated in the fruit, the enzymatic hydrolysis produces hydroxytyrosol, tyrosol, demethyloleuropein and secoiridoide glycoside, arising from the degradation of complex polyphenols.

\section{Olive Oil Aroma Biogenesis}

Virgin olive oil has unique characteristics which results from its fatty acid composition and the presence of minor compounds including volatile and phenolic compounds. Some of these are biosynthesized during the olive fruit ripening, especially during the climacteric period. Some volatile compounds are still present in the fruit belonging from the lipids or amino acids metabolism [9, 10]. However, the majority of the olive aroma derives from enzymatic oxidation of the fatty acids, linoleic and linolenic [11].

Saturated aldehydes having carbon number comprised between 7 and 12 are the major responsible of VOO aroma. This class of volatile compounds is usually present at concentrations of 50 and $75 \%$ of the total volatiles in unripe and fully mature olive drupes, respectively [12] [Table 1].

\begin{tabular}{|c|c|c|}
\hline Compound & Sniffing & MDS (multidimensional scaling) \\
\hline Methyl acetate & & Green (nuts) \\
\hline Octene & Solvent-like & Green (grass) \\
\hline Ethyl acetate & Sweet, aromatic & Slightly bitter/pungent \\
\hline 2-Butanone & Fragrant, pleasant & Tomato, apple \\
\hline 3-Methylbutanal & Sweet, fruity & Apple \\
\hline 1,3-Hexadien-5-yne & & Green (green olives) \\
\hline An alcohol & Sweet, apple & Other ripe fruit \\
\hline Ethylfuran & Sweet & Sweet \\
\hline Ethyl propanoate & Sweet, strawberry, apple & Sweet \\
\hline An alcohol hydrocarbon & Pungent, acid fruit & \\
\hline 3-Pentanone & Sweet & Sweet \\
\hline 4-Methylpentan-2-one & Sweet & Green \\
\hline Pent-1-en-3-one & Sweet, strawberry & Sweet \\
\hline 2-Methylbut-2-enal & Solvent -like & Ripe fruit (olives, dry wood) \\
\hline 2-Methylbut-3-enol & & Slightly bitter \\
\hline A hydrocarbon & Sweet, apple & Sweet \\
\hline Methylbenzene & Glue, solvent-like & Overripe fruit \\
\hline Butyl acetate & Green, pungent, sweet & Sweet \\
\hline Hexanal & Green, apple & Sweet \\
\hline A hydrocarbon & Sweet, aromatic & Sweet \\
\hline $\begin{array}{l}\text { 2-Methylbutyl } \\
\text { propanoate }\end{array}$ & Aromatic, ketone & Olive, apple \\
\hline 2-Methyl-1-propanol & Ethyl acetate-like & Green \\
\hline (E)-2-Pentenal & Green, apple & Ripe fruit (soft fruit) \\
\hline An alcohol & Greasy & Undesirable (rancid) \\
\hline (Z)-2-Pentenal & Green, pleasant & Overripe fruit \\
\hline Ethylbenzene & Strong & Bitter taste (dried green herbs) \\
\hline (E)-3-Hexenal & Artichoke, green & Artichoke \\
\hline (Z)-3-Hexenal & Green, green leaves & Green \\
\hline 1-Penten-3-ol & Wet earth & Undesirable \\
\hline 3-Methylbutyl acetate & Banana & Slightly fruity \\
\hline Heptan-2-one & Fruity & Ripe fruit \\
\hline (E)-2-Hexenal & Bitter almonds & Bitter \\
\hline (Z)-2-Hexenal & Fruity, almonds & Almond odor, bitter taste \\
\hline 2-Methylbutan-1-ol & Fish oil & \\
\hline $\begin{array}{l}\text { 3-Methy1-2-butenyl } \\
\text { acetate }\end{array}$ & Putty-like unpleasant & Ripe fruit \\
\hline
\end{tabular}




\begin{tabular}{|l|l|l|}
\hline Dodecene & & Slightly bitter -taste \\
\hline Pentan-1-ol & Pungent & Ripe fruit \\
\hline Ethenylbenzene & & Fruity \\
\hline 3-Methyl-butanol & & Undesirable (yeast) \\
\hline Hexyl acetate & Sweet, fruity & Green (grassy) \\
\hline A ketone & Fruity, mushroom -like & Green \\
\hline Octan-2-one & Moldy & Undesirable \\
\hline $\begin{array}{l}\text { 3-(4-rnethyl-3- } \\
\text { pentertyl)Furan }\end{array}$ & Paint-like strong & Overripe \\
\hline 3-Hexenyl acetate & $\begin{array}{l}\text { Green banana, green } \\
\text { leaves }\end{array}$ & Green \\
\hline (Z)-2-Penten-1-ol & Banana & Green (grass) \\
\hline 6-Methyl-5-heptert-2-one & Fruity & Bitter taste (dried green herbs) \\
\hline Nonan-2-one & Fruity & Apple \\
\hline Hexan-1-ol & Fruity, aromatic, soft & $\begin{array}{l}\text { Rough mouthfeel and } \\
\text { rancid odor }\end{array}$ \\
\hline (E)-3-Hexen-1-ol & & Green leaf, nuts \\
\hline 2,4-Hexadienal & & Ripe fruit \\
\hline (E)-2-Hexen-1-ol & Green, grassy & Green (cut green grassy) \\
\hline Acetic acid & & Undesirable \\
\hline Methyl decanoate & Fresh & Green leaf, nuts \\
\hline Tridecene & & Bitter (almond) \\
\hline (Z)-3-Hexen-1-ol & Banana & Green banana \\
\hline
\end{tabular}

Aliphatic and aromatic hydrocarbons, aliphatic and triterpenic alcohols, aldehydes, ketones, ethers, esters and furan derivatives are present in the VOO aroma. The most abundant individual compounds are hexanal, trans-2-hexenal, hexanol and 3-methylbutanol [11, 12]. Among the volatile compounds, those attributed to the sensory note described as "green" are C6 aliphatic compounds, hexanal, cis-3-hexenal, trans-2-hexenal, hexanol, cis-3-hexenol, trans-2-hexen-1-ol and the corresponding esters [13].

The following volatile compounds account to circa $80 \%$ of the total headspace composition:

- C6 aldehydes (hexanal, cis-3-hexanal, trans-2-hexanal);

- Alcohols (hexanol, cis-3-hexenol, trans-2-hexenol);

- Esters (hexyl acetate, cis-3-hexenyl acetate [9,11,14].

The Lipoxygenase (LOX) pathway is a prominent enzymatic route for olive oil aroma generation, and involves a variety of enzymes acting on unsaturated fatty acids. LOX is activated when the olive drupe is crushed, which is the first step of olive oil extraction process. During olive crushing and the next malaxation phase, the typical olive oil aroma is produced [14]. The LOX pathway involves the activity of four enzymes, namely lipoxygenase, hydroperoxide lyase, alcohol dehydrogenase and alcohol acyltransferase [9, 13, 14].

When the integrity of olive drupe is compromised, 9- and 13-hydroperoxides of linolenic and linoleic acids are produced $[4,13]$.

The hydroperoxide lyase produces aldehydes, subsequently reduced in alcohols by the alcohol dehydrogenase. These compounds are then esterified by the alcohol acetyltransferase to produce esters $[9,14]$.
The 13-hydroperoxide undergoes an enzymatic scission by the hydroperoxide lyase, with the subsequent production of hexanal and cis-3-hexenal. The hydroperoxide lyase found in the olive pulp is highly specific for 13-hydroperoxides $[9,15]$. The 13-hydroperoxide formed from linolenic acid is rapidly reduced to cis-3-hexenol, from which cis-3-hexenyl acetate is produced. Alternatively, it is isomerised to form trans-2hexenal, which is then reduced to trans-2-hexenal by following a different biosynthetic route [4].

\section{Influence of Botanical and Agronomical Factors}

\section{Variety}

Olive cultivar has been reported as one of the most crucial factors responsible for olive oil flavour variability [16]. Virgin olive oil extracted in the same conditions can show dramatic differences in their composition and sensory characteristics because they are extracted from different varieties [17] [Figure 1]. Volatile compounds having six carbon atoms, especially trans-2-hexenal, have been regarded as good indicators for differentiation of single-variety olive oils [18].

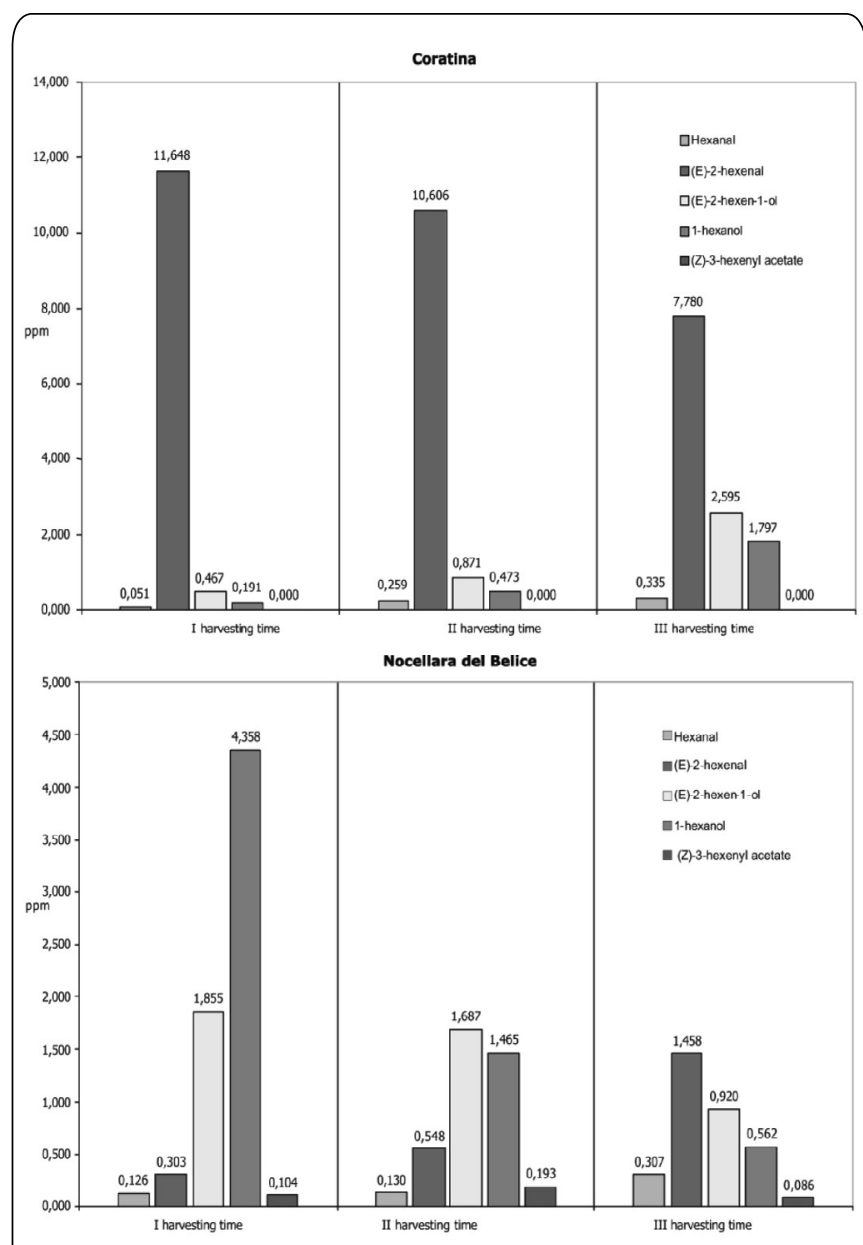

Figure 1: Variation of some volatile compounds in VOOs obtained from single olive varieties, from olives harvested at longer maturation degrees (adapted from Benincasa et al. [5]).

The olive variety also influences the concentration of phenolic compounds, whereas the major influence was 
attributed to the olive ripening degrees and environmental conditions [19].

Dhifi et al. [20] reported the concentration of aroma compounds in VOO extracted from four Tunisian olive cultivars, i.e. Chetoui, Chemlali, Chemchali, Oueslati. A wide range was described depending on the variety, with cv. Chemchali showing $59.7 \mu \mathrm{g} / \mathrm{mL}$, while cv. Oueslati had the lowest concentration, i.e. $15.7 \mu \mathrm{g} / \mathrm{ml}$.

Other researchers have compared Tunisian cultivars with Italian ones, reporting that cv. Chetoui is characterized by a low content of $\mathrm{C} 6$ aldehydes, responsible of the sensory notes "green" and "fruity" [21]. Ranalli et al. [22] reported concentrations of 230.4,381.1 and $401.7 \mathrm{ppm}$ total volatiles for the Italian cultivars Cipressino, Cassanese e Leccino, respectively.

According to Tura et al. [16], the major volatile compounds affected by the olive cultivars are the following ones: ethanol, 2-methyl propanol, pentanol, cis-2-penten-1ol, cis-3-hexenol and octanol. They have been associated to the following sensory notes, respectively: floral, banana, apple, walnut, hay, butter, sweet and fruity [16].

\section{Ripening degree}

The oil accumulation in the olive drupe starts about 5 weeks after anthesis, and its major increase is between 60 and 120 days after flowering [9]. During the olive maturation phase, the fruit undergoes several modifications: exocarp color change from green to violet-black, lower fruit consistency, higher free acidity, lower pigment concentration, phenolic compounds concentration, which concentration is maximum when the darkening phase starts and a continuous decrease follows [23].

Fatty acid composition is quantitatively affected by two main factors, i.e. the olive variety used and the ripening stage at which the olives were harvested. The differences in the fatty acid profiles in different varieties were partially related to the biosynthesis of fatty acids during ripening. Specifically, stearoyl-acyl carrier protein (Stearoyl-ACP) is obtained from Acetyl-CoA by the action of an enzymatic complex called fatty acid synthase I and III (FAS I/III) followed by FAS II. Stearoyl-ACP is desaturated to oleoyl-ACP (C18:1-ACP) by the stearoyl-ACP 9-desaturase, which is highly active in the plastids. As growth proceeds, desaturase transcripts accumulate at higher levels, and the high transcription rate remains up to 28 weeks after flowering. This transcription pattern is observed during fruit development parallels with the synthesis of oleic acid in olives [24].

The fatty acid composition of VOOs is known to depend mainly on genetic factors, i.e. olive cultivar, and therefore several investigations have tried to study whether it is possible to discriminate the $\mathrm{VOO}$ variety based on the fatty acid profile, e.g. in Spanish cultivars Arbequina, Hojiblanca and Picual, which are among the most important ones worldwide. The authors reported a very good discrimination power by Partial Least Square, whereas they also add the phenolic composition and not just fatty acid profile [25].

The oil quality is strictly linked to the fruit physiological conditions, as the progress of the ripening phase cause higher production of free acidity, with a contemporary decrease of phenolic compounds responsible for the bitter-pungent notes [26] [Figure 2].

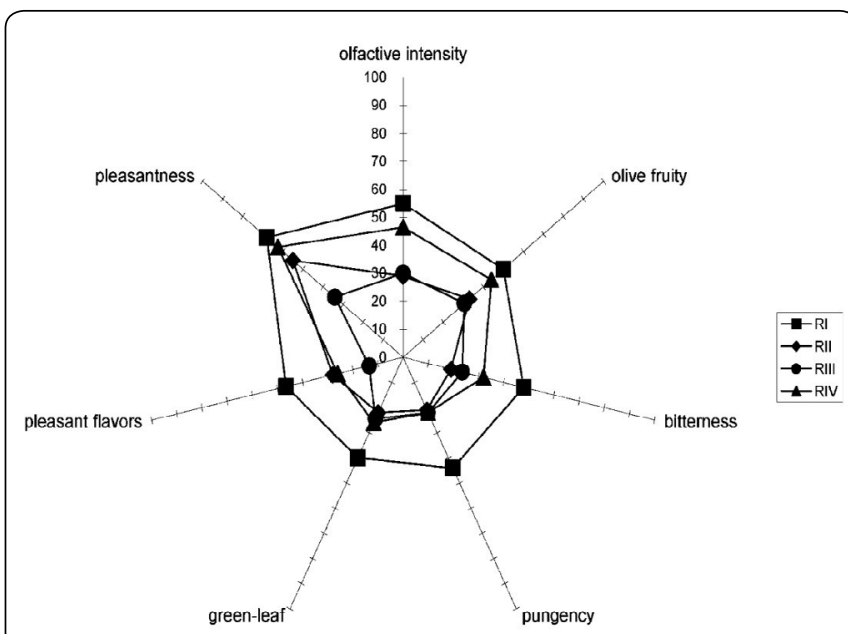

Figure 2: Sensory profile of VOOs obtained from cv. Nostrana di Brisighella at increasing ripening degrees (adapted from Rotondi et al. [30]).

To define the olive drupe maturity degree, the most common method applied rely on the observation of the fruit skin and pulp colors, according to the method reported by Uceda and Frias [27]. It describes seven maturity states of the fruit (0, bright green skin; 1 , green-yellowish skin; 2 , green skin with reddish spots; 3 , reddish-brown skin; 4, black skin with white flesh; 5 , black skin with $<50 \%$ purple flesh; 6 , black skin with $\geq 50$ and $>100 \%$ purple flesh; and 7 , black skin and purple flesh), and the agronomist or farmer randomly takes 100 fruits from the field, then segregates them into groups and calculates the number of each fruit per group color, by using a color chart.

Olive maturity degree also influences the generation of aroma compounds, being the maximum concentration observed when the fruit starts the color changing phase, and followed by a continuous decrease $[12,17,28]$.

It is known that at higher ripening stages, the aromatic note describes as "fruity-grassy" is significantly lower than oils obtained from greener olives. The sensory notes of bitterpungent and other positive aromatic notes decrease with increasing ripening degree [23]. The increase in olive maturity leads to the increase in 1-penten-3-ol. The compounds hexanal, trans-3-hexenol, cis-3-hexen-1-ol and cis-2-hexenol were reported to have high selectivity for unripe olives. The intermediate maturation phase was characterised using hexyl acetate, while over-ripe drupes can be discriminated using trans-3-hexenal, cis-2-hexenal, trans-3-hexenol and cis-3hexenol [28].

Some triterpenic alcohols were be proposed as markers of fruit maturity. Sakouhi et al. [29] demonstrated that triterpenic alcohols and 4-monomethyl sterols concentrations in the olive fruit increase from 18 to $30 \%$ during ripening of the cv. Picholine.

A negative correlation $\left(\mathrm{r}^{2}=-0.88\right)$ between the drupe ripening degree and phenolic content in $\mathrm{VOO}$ was also 
reported by Rotondi et al. [30]. Gomez-Rico et al. [31] studied the combined effect of maturity and irrigation on the phenolic and volatile compounds of VOOs obtained from cv. Cornicabra. They reported that complex phenolics undergo the major changes, with a stronger loss depending on the irrigation. This was linked to the higher activity of L-phenylalanine ammonia-lyase in condition of plant stress.

During drupe maturation, an increase of the triterpenic acid 24-methylene-cycloartenol has been reported, with a contemporary decrease in cycloartenol. Also, sterol compounds undergo significant changes, e.g. increasing the sterol $\Delta 5$ avenasterol and decrease of $\beta$-sitosterol [32].

A recent paper reported that trans-2-hexenal concentration decreased as maturity progressed, for some Turkish varieties [33]. The authors also described that the concentration of all aroma compounds, except trans-2-hexenol and hexyl acetate, decreased with the degree of ripeness, and that altitude plays a significant but less intense effect on the volatile composition [33].

The olive fly (Bactrocera olea) is the most important biotic stress affecting the drupe quality and the resulting olive oil. VOO produced from fruits attacked by the olive fruits has higher free acidity value and peroxide number, different fatty acid composition and concentration of phenolic compounds [34]. Significant differences from the sensory panel scores of these oils were also reported [35]. The sensory profile of such VOOs had lower scores when the olives are harvested at full maturity stage, with the appearance or increase of negative sensory notes such as fusty, mouldy and winey-vinegary. Olive fly attacks cause a significant decrease of the attribute "pungent-bitter", due to the hydrolysis of complex phenolic compounds caused by the insect damages [36].

\section{Environmental and climatic conditions}

Olive is a xerophyte plant, and it needs a mild climate, with no sudden temperature changes below $-8{ }^{\circ} \mathrm{C}$, and with its full potential between 22 and $32{ }^{\circ} \mathrm{C}$. At higher latitudes, an increase in oleic acid content and a higher ratio of unsaturated to saturated fatty acids has been reported. The environmental conditions, slowing down the ripening of fruit, can diminish the activity of certain hydrolytic enzymes which act on phenols [37].

Several studies have been conducted on the effect of soil salinity and irrigation water on the production and VOO fatty acid profile, but still scarce and contradictory results have been published so far in relation to the effects on their organoleptic characteristics. For example, Ahmed et al. [38] reported an increase in the concentration of total phenolic compounds, especially tyrosol, hydroxytyrosol and vanillic acid, and a reduction in the ratio unsaturated to saturated fatty acids, with increasing salinity of the water.

The influence of the organic agronomical practice on the VOO composition and flavour characteristics has been scarcely investigated. In particular, Ninfali et al. [39] reported higher concentrations of phenolic compounds from organic than non-organic VOOs in the first year of the experiment, while this difference was not significant over the second and third year. From the panel test, organic VOOs have shown a stronger note of "hay" and "artichoke". Non-organic oils presented more markedly floral notes of "fresh grass" and "fruity". The results are not consistent, however, in all the years, and it is strongly influenced by climate change over the years [39].

Water deficit represents one of the main environmental factors limiting its production potential, caused by the decrease of the fruit endogenous esterase's. Rainfall has prominent influence with respect to the environmental temperature, and some volatile compounds such as hexanal and isobutyl acetate were negatively correlated to the rainfall [18].

The amount of water applied to the orchard can modify the fatty acids ratio, with a higher concentration of monounsaturated and polyunsaturated fatty acids at higher water volumes applied. Salas et al. [40] reported a clear inverse correlation between the amount of irrigation water applied and the intensity of the bitter note in the VOO. Also Gomez-Rico et al. [31] confirmed previous findings piloting three water management systems: no added water, fully water addition, controlled deficit irrigation using by the method proposed by FAO [Figure 3].
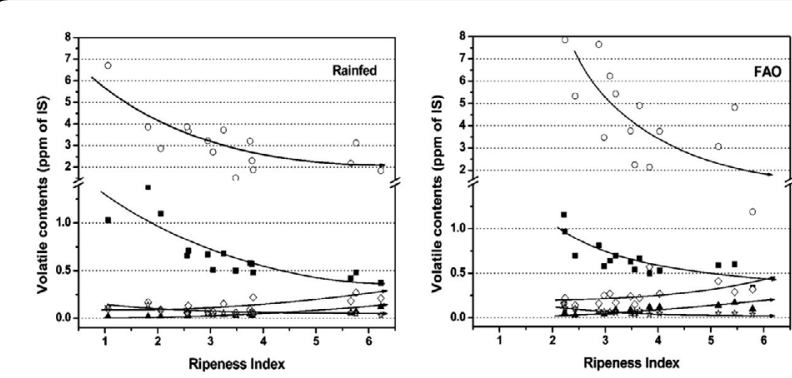

Figure 3: Evolution of main volatile compounds in VOOs under rain-fed conditions and FAO irrigation strategy during fruit ripening (crop season 2003/2004). White circle: trans-2-hexenal; square: hexanal; white rhombus: cis3-hexenl-1-ol; triangle: hexan-1-ol; black rhombus: trans-2-hexen-1ol (adapted from Gomez-Rico et al. [31]).

Greater water availability seems to also affect $\mathrm{VOO}$ volatile compounds concentration, especially trans-2-hexenal, cis-3hexen-1-ol and hexanol, in the sense of their decrease with increasing irrigation volume. Some other volatile compounds were not reported to be subject to variations, such as 1-penten3-one and 1-penten-3-ol, as they were linked more to seasonal factors and ripening degree than the irrigation practices [31]. In subsequent studies, the same authors [41] verified that the hexanal also shows an inverse variation of its concentration with increasing water availability [Figure 4].

Opposite conclusions were reported by Stefanoudaki et al. [42], where lower values of 1-penten-3-ol and 1-penten-3-one found as a result of the irrigation, for $\mathrm{cv}$. Leccino but not for $\mathrm{cv}$. Cornicabra, in which there were no significant modifications. It was reported a decrease in concentration of the total volatile compounds in olive oils irrigated. The C5 compounds undergo a drastic decrease but, in contrast to other authors, no increases were reported for $\mathrm{C} 6$ compounds.

Cornicabra variety is of great importance in Spain because comprises about $10-12 \%$ of its production, and is one of the 
most important after the Spanish Picual variety. Cornicabra variety shows late maturation than other olive varieties with the pulp and the skin being greener also at high maturity stage. This suggests that the ripening has a different effect on olive oil composition and quality, compared to other olive oil varieties. This aspect is very interesting and needs further research to evaluate the specific effect of ripening on different varieties.

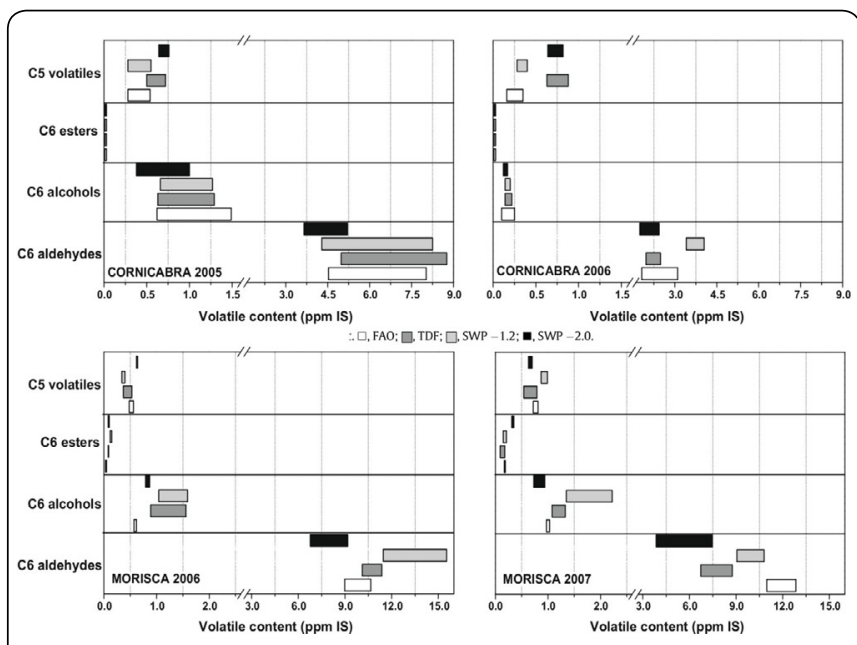

Figure 4: Concentration of volatile compounds C5 and C6 derived from the lipoxygenase pathway in VOOs from cv. Cornicabra and Morisca, depending on the water strategy applied on the orchard (ripening degree: 3-3.5) (adapted from Gomez-Rico et al. [41]).

The irrigation strategy has been reported to be more effective than the total amount of rainfall during the year, as the concentration of phenolic compounds depends on the hydric stress [31, 42]. Also fatty acids undergo modifications, with an increase in the monounsaturated, i.e. oleic acid, to polyunsaturated fatty acids [43]. The sensory evaluation of VOOs from irrigated orchards seem to show lower intensity of the attributes fruity, bitter and pungent [42].

A recent research paper reported on the influence of climate, soil composition, agricultural practices (fertilization and irrigation) and variety on the compounds responsible for the flavour of extra VOO (volatiles and phenols) and how these compounds can explain the differences in chemical profiles by geographical origin, cultivar and fruit ripeness stage [44]. The authors reported that the soil and the climate of the Chilean regions have much more influence than cultivars on the concentration of sensory quality compounds. The difference in latitude between the orchards increases the importance of the geographical origin on the virgin olive oil chemical composition, while at the same time, full irrigation decreases the influence of the olive variety, which seems to be an opposite trend with respect to the Mediterranean cultivation area [44].

\section{Geographic location}

Studies by Montedoro et al. [45] reported the possibility to discriminate VOOs from different Italian regions through the analysis of volatile compounds. VOO from Italy were reported as richer in $\mathrm{C} 6$ aldehydes and lower in fruity esters then Moroccan oils [46]. Vichi et al. [47] did not observe any difference in the concentration of $\mathrm{C} 5$ compounds in different varieties, thus suggested that these volatiles might be influenced mainly by the geographical location. VOO from Chetoui varieties from Tunisia had different profiles according to different cultivation sites, particularly for the fatty acid profile and phenolic compounds concentration [48].

Many authors have tried to discriminate VOO according to their geographical origin. Issaoui et al. [49] studied the effect of the difference in altitude, latitude and climatic conditions (north and south of Tunisia) in the cv. Chemlali and Chetoui.,VOOs from the northern regions of Tunisia contain mainly the following volatile compounds: cis-3hexenyl acetate, cis- $\beta$-ocimene, hexyl acetate and cis-cis- $\alpha$ farnesene.

There seems to be a general agreement about some volatile compounds which are dependent on the geographical area of origin, i.e. hexanal, hexanol, trans-2-hexenal, cis-3-hexenal, trans-3-hexenol, cis-3-hexenol and trans-2-hexenol [47].

The application of cluster analysis based on "green" volatile compounds allowed to group 39 varieties with the aim to find markers for volatile compounds that could discriminate oils coming from different areas [50]. The following compounds were described as the most important ones for Italian and Spanish VOOs: 2-methylbutyl acetate, 2-methyl-4-pentenal and hexanol. For the distinction of Italian oils by the Greek ones, pentane-3-one, cis-3-hexenal and a hydrocarbon were identified. Regression analysis allowed to classify oils derived from macro-areas of origin (Spain, Italy and Greece) by the following volatile compounds: hexyl acetate, ethyl benzene, 2-methyl-4-pentenal, ethyl furan, trans-2-pentenal, 1,2,3-trimethylbenzene, 3-methyl butanol and a hydrocarbon [50].

\section{Harvesting systems}

The olive harvesting system exerts a dramatic influence on the final VOO flavour. Oils derived from olives picked up from the soil are qualitatively poorer, and both the chemical parameters (acidity, peroxides, UV) and the organoleptic characteristics might be affected. With the protracted contact times of the olives with the ground, trans-2-hexenal and cis-3hexenyl acetate ("green notes") concentration decrease, and the concentration of hydrocarbons, acetic acid and other carbonyl compounds increase, with consequent negative organoleptic notes [18].

The mechanical harvesting of the olives seems not to be of significant influence on the chemical composition of VOOs. The olive harvesting needs to be performed trying not to damage the drupe exocarp and mesocarp, to prevent spontaneous fermentation and mold growth that would result in quality deterioration and occurrence of defects oil.

Tura et al. [16] reported the importance of the degree of maturity on the content in phenolic compounds and volatile, but also fruit storage can result in a change of the flavour profile, due to the reduction of esters and aldehydes responsible for the positive notes [46].

In single-variety oils, cv. Correggiolo obtained from olives with maturation index (expressed as an index of Jaén) of less than 2.5, with the increase of time of storage of olives there is a general decrease of the content of volatile substances, 
in particular of those responsible for the sensations of fruity, almond, green notes (trans-2-hexenal, hexanal, cis-3hexenol) and sweet notes (1-penten-3-one, 3-pentanone), simultaneously with an increase of compounds which are associated notes odorous (3-methyl-1-butanol, 2-methyl-1propanol).

\section{Influence of the Olive Oil Extraction System}

\section{Olive crushing}

The majority of VOO aroma compounds are produced during the crushing phase, when the enzymatic oxidation of linoleic and linolenic acid takes place [21]. The type of milling system thus influences the final oil quality, by changing the quali-quantitative composition of some minor constituents, with consequent influence on the organoleptic properties and product stability.

There are two major types or olive crushers: granite stone mill and metallic crusher. This letter can be divided into two types, i.e. disc and hammer crushers. The hammer crushers have stronger effects and cause a strong emulsification on the olive paste and an increase of its temperature, due to friction phenomena, with disadvantages on the yield and on the oil quality. It should be also noted that the stone mill is a discontinuous system, while the metallic crusher system is used in continuous and therefore their performance in terms of processed olive is much higher.

VOO obtained using stone crusher is more aromatic and harmonic than oils obtained using the other two types of crushers. The disc crusher, however, leads to the formation of oil richer in phenolic compounds, more bitter and stable to lipid oxidation over storage [51].

Olias et al. [13], on the contrary, reported that stone mill leads to higher concentration of volatile compounds than metallic crushers, e.g. trans-2-hexenal, hexanal and cis3-hexenol. For cv. Coratina and Oliarola, Servili et al. [52] reported that disc crusher cause higher concentrations of $\mathrm{C} 6$ aldehydes and some esters such as hexyl acetate, 3-hexenyl acetate and cis-4-hexenyl acetate, than hammer crusher. Therefore, the olive milling technology is the first parameter to be considered for the setting of the best extracting conditions.

\section{Malaxation}

The malaxation is a fundamental stage in VOO extraction, which involves the continuous slowly mixing of the olive paste to aggregate the oil/water emulsion. Malaxation time and temperature are parameters that are usually controlled by the industry during VOO extraction process, which can potentially affect the sensory profile and composition of the final product [46] [Figure 5]. The factor "malaxation time" was reported to be positively correlated to the total content of volatile compounds, but negatively correlated with the concentration of total phenolics [22]. Longer malaxation times promote the accumulation of alcohols and C5 other compounds, particularly hexanal. The temperature increase speeds up the activity of oxidative enzymes such as polyphenol oxidase, lipoxygenase and peroxidase [11].

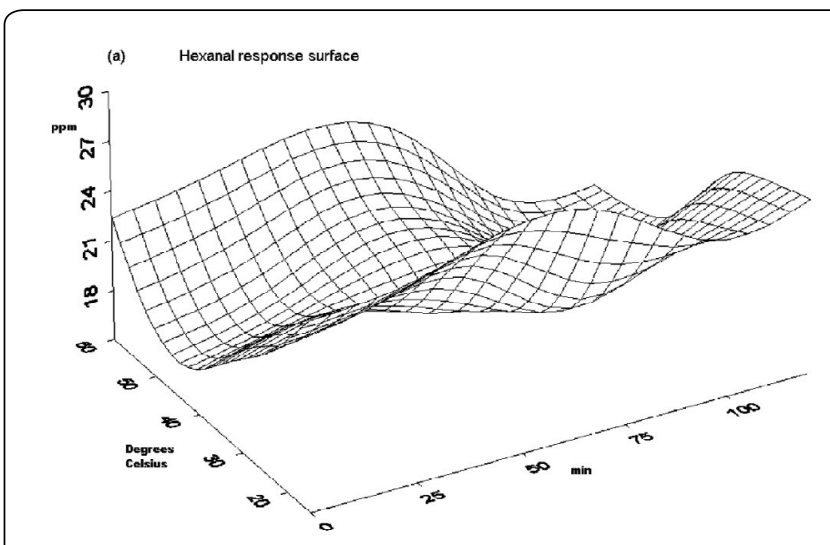

Figure 5: Malaxation time-temperature response surface for hexanal generation (adapted from Kalua et al. [10]).

However, there is a loss of volatile compounds when high malaxation temperatures are applied, attributed to the enzymes deactivation, especially hydroperoxide lyase [14]. Indeed, the increase of malaxation temperature causes a considerable decrease of C6 compounds, cis-3-hexenol and C5 metabolites, as well as an increase in hexanol and trans-2-hexen-1-ol and a loss of the bitter-pungent notes [53].

Angerosa et al. [53] studied the combined effect of malaxation temperature and time on the VOO aroma from cv. Coratina and Frantoio, and highlighting that malaxation negatively affects the total amount of secoiridoids. Some phenolic compounds, such as tyrosol, seem not undergo major changes as affected by the malaxation time, but they are only affected by the temperature adopted. The opposite trend was reported for the phenolic compound 3,4-DHPEADEDA, for which the maximum concentration was obtained at short malaxation time. The loss of phenolic compounds during malaxation is due to the enzymatic oxidation of the secoiridoid derivatives, catalysed by polyphenol oxidase and peroxidase [54].

The hydrolysis of complex phenols (oleuropein, dimethyl oleuropein, ligstroside, verbascoside, rutin and luteolin7-glucoside) by the endogenous glucosidase results in the production of aglycones and simple phenols, which dissolve appreciably in the oil phase, while the repartition of unhydrolysed glycosidic phenols is more toward the aqueous phase [55].

The following compounds were proposed as markers for malaxation temperature: 1-penten-3-ol, cis-3-hexenal and octane, which were proposed as markers to discriminate malaxation temperatures of 15,30 and $35^{\circ} \mathrm{C}$, respectively [10] [Table 2]. The concentration of oxygen in the paste headspace during the malaxation process can be manipulated to achieve significant modification of the VOO aroma, in relation to the desired characteristics and industrial needs [54].

\section{Extraction phase}

The separation of the liquid phase and solid particles from the olive paste is usually performed using two major systems, i.e. pressure or centrifugation. The subsequent step involves the separation of the oil from the oil-water mixture, which is performed by centrifugation. 
Table 2: Discrimination of individual malaxation times and temperatures based on VOO volatile and phenolic compounds (from Kalua et al. [10]).

\begin{tabular}{|c|c|}
\hline \multicolumn{2}{|r|}{ Discriminating Variables } \\
\hline \multicolumn{2}{|l|}{ Time } \\
\hline $30 \mathrm{~min}$ & Hexanal, 3,4-DHPEA-DEDA ${ }^{\mathrm{a}}$, and $\mathrm{FFA}^{\mathrm{b}}$ \\
\hline $60 \mathrm{~min}$ & $\begin{array}{l}\text { Z-2-Penten-1-ol, Hexanal, Acetoxypinoresional, and } \\
\text { FFA }^{\text {b }}\end{array}$ \\
\hline $90 \mathrm{~min}$ & Z-2-Penten-1-ol, Acetoxypinoresinol, and yield \\
\hline $120 \min$ & Z-2-Penten-1-ol, Acetoxypinoresinol, and yield \\
\hline \multicolumn{2}{|c|}{ Temperature } \\
\hline $15^{\circ} \mathrm{C}$ & 1-Penten-3-ol, E-2 Hexenal, and Vanillic acid \\
\hline $30^{\circ} \mathrm{C}$ & E-2-Hexenal \\
\hline $45^{\circ} \mathrm{C}$ & Tyrosol and FFA ${ }^{\mathrm{b}}$ \\
\hline $60{ }^{\circ} \mathrm{C}$ & Hexanal, Octane, and 3,4-DHPEA-DEDA ${ }^{a}$ \\
\hline
\end{tabular}

a3,4-Dihydroyphenyl ethyl alcohol-decarboxymethyl elenolic acid dialdehyde.

${ }^{\mathrm{b}}$ Free fatty acid expressed as percent of oleic acid.

When separation systems based on pressure area applied, the VOOs tend to be higher in the fruity sensory score and with a high concentration of volatile alcohol, but possible fermentation and/or degradation phenomena can take place, with consequent sensory defects [11].

Differences in phenolic content in the oils was also observed between the conventional centrifugal ("three-phase") and the "two-phase". The latter does not involve the addition of water (or very little amounts), which results in a lower dilution of hydrophilic phenolic compounds such as orthodiphenols and hydroxytyrosol.

The use of two phases centrifuge, compared to the threephase one, allows the production of VOOs with higher concentrations of trans-2-hexenal and greater total aromatic content, but with lower concentration of pigments, aliphatic and triterpene alcohols, sterols and waxes [56]. The use of the three-phases centrifuge causes decrease in the content of $\mathrm{C} 6$ aldehydes, hexanol and trans-2-hexenol compared with the pressure extraction, probably because of the addition of hot water [18]. Incorrect or improper management of the pressure extraction system may lead to olive paste fermentation that and the subsequent off-flavours [57].

\section{Olive oil filtration and storage}

VOO filtration may have important effects on its sensory properties and shelf life. Unfiltered oils contain a certain amount of water (from $2 \mathrm{~g} / \mathrm{kg}$ to $4 \mathrm{~g} / \mathrm{kg}$ ) dispersed in microdroplets present in dispersion as part of the colloidal system of the fruit. The filtration might influence the bitter-pungent sensory note, caused by the hydrolysis of phenolic compounds. It has been recently reported that the industrial-scale filtration of highly bitter-pungent extra $\mathrm{VOO}$ has influence on the release of key aroma compounds in the product after storage, and therefore the filtration process should be also regarded as one of the possible parameter influencing VOO flavour [58].

VOO quality, similarly to other vegetable oils, is affected by the storage conditions. Oxygen and light exposure, high temperatures and presence of metal ions as trace can accelerate the lipid oxidation and then shorten the shelf life of the product. The stability of VOO depends on its fatty acid composition, in particular by the ratio of the oleic to linoleic acid, and presence of minor compounds such as $\alpha$-tocopherol, carotenoids, squalene and phenolics [19].

Among the saturated aldehydes, nonanal and hexanal suffer a sharp increase as oxidation rate increases. Some authors suggested to use the relationship hexanal/nonanal headspace concentration as an indicator of the oxidation status, while others reported that trans-2-heptenal is associated with the perception of rancid defect $[46,47]$. Trans-2-Hexenal is the most abundant volatile compound present in VOOs, and it undergoes continuous decrease over storage. The profile of phenolic compounds in VOO can continuously change over storage, as hydrolytic processes take place on complex forms of oleuropein derivatives, as well as oxidation of ortho-diphenols [18]. It is known, in fact, that prolonged storage cause the increase in simple phenolic compounds such as tyrosol and hydroxytyrosol with a contemporary decrease in complex forms and an effect also on the sensory aspect.

The type of container where the oil is stored was also reported to influence the lipid oxidation status and the chemical composition of VOOs under normal retail storage conditions [59], and therefore this parameter should be carefully considered to avoid quality loss over storage.

\section{Olive oil aromatisation}

A niche market for olive oil involves the flavouring by the addition of natural aromatic agents, particularly spices and herbs. This is usually done to give extra flavour and new or unexpected aromas, and it is typical for some countries of the Mediterranean area. For example, the aromatisation of olive oil using red dried chili pepper by the traditional infusion method was recently studied by Caporaso et al. [60]. The authors reported that the addition of dried chili pepper caused a significant increase in hexanal, related to oxidation processes. 2-methylbutanal, 3-methylbutanal and 6-methyl5-hepten-2-one were also detected in flavoured VOO and derived from chili as degradation products of the drying process. Apart of the volatile compounds, also a considerable amount of capsaicinoids were released in the flavoured oils, with consequent spicy and pungent taste [60].

\section{Sensory Description of VOO Aroma Compounds}

The most abundant volatile compounds in $\mathrm{VOO}$ are aldehydes and $\mathrm{C} 6$ alcohols, which were related to the sensory note of sweetness. Aldehydes and C5 alcohols contribute to other positive attributes of VOOs. The odour threshold of VOO volatiles is dramatically different depending on their chemical structure and physio-chemical characteristics. For example, cis-3-hexenal, which gives the typical odour of freshly cut grass and despite its low concentration, greatly contributes to the aroma as it has a very low odour threshold. On the contrary, trans-2-hexenal has much higher perception threshold and it contributes minimally to the final VOO 
aroma, despite of being the most abundant volatile in the oil [46].

Trans-2-Hexenal and trans-2-hexenol have been associated to VOOs obtained from olives in good conditions. The following compounds contribute mostly to the "green note": cis-3-hexenal, cis-3-hexenol (grass and banana) and cis-3-hexenyl acetate (fruity and green leaves). Important in defining the complex flavour are also trans-2-hexenal, hexanol and trans-3-hexenol. Hexyl acetate contributes to perceptions of fruity and sweet, while hexanal is responsible for the "green" and apple note. This latter compound is not only produced during the lipoxygenase pathway, but is also generated from lipid auto-oxidation, together with other oxidation products [22]. cis-3-Hexenol has been associated to the bitter note, as well as the attributes of "apple", "tomato", "vegetable bitter", "grass" and "fruity olive oil", along with some C5 alcohols (cis-2-penten-1-ol and 1-penten-3-ol) and trans-2-hexenal [61]. Other compounds such as toluene, octane, octene and 3-methyl butanol arise from different routes from the LOX pathway, and are not related to positive sensory attributes of VOO.

The "fruity" note of VOO was positively correlated with the cis-3-hexenol and negatively correlated with 3-pentanone. The "ripe fruit" sensory description is correlated with 3-pentanone, while "leaf" is correlates positively with hexyl acetate, 1-penten-3-ol, cis-2-penten-1-ol and negatively correlated with hexanol, associated with the "grass" note. The note of "almond" correlates positively with the hexyl acetate, 1-penten-3-ol and cis-2-penten-1-ol and negatively with the hexanol; cis-3-hexenol correlates positively with the scent of "tomato" and "bitter vegetable"; 3-pentanone correlates negatively with the attributes of "bitter", "spicy", "tomato" and "vegetable bitter" [11].

The attributes of bitter and spicy oil are due to the presence of phenolic compounds. The bitter taste is attributed to compounds aglycone form the dialdehydic form of the decarboxymethyl oleuropein, and other forms of the oleuropein aglycone; the "pungent" note has been attributed to the aglycone form the dialdehydic of the decarboxymethyl ligstroside [62].

The sensory defect of "winey-vinegary" is associated with fermentative processes especially from Lactobacillus, when olives are left on the ground for prolonged times. The consequent production of acetic acid is a marker of olives collected from the ground. A high concentration of acetic acid and octane was correlated to the defect of "reheating", a consequence of the activity of Enterobacteriaceae the genus Aerobacter and Escherichia during the first days the permanence of the olives to the ground. The genera Pseudomonas, Clostridium and Serratia appear if the olives are left in bags for a long time after harvesting. The activity of these microorganisms results in the presence of some volatile compounds at high concentrations. The defect of "mouldhumidity" has been correlated to the presence of 3-methyl1-butanol. Other compounds that are important in defining the overall aroma oil, were reported in low concentrations in the olive paste: the hexyl acetate, characterized by the smell fruity and marker of good quality oils, it does not contribute to the aroma of the paste due to its high perception threshold $(\sim 1: 04 \mathrm{mg} / \mathrm{kg})$ and low concentration in the paste (maximum at $1.00 \mathrm{mg} / \mathrm{kg}$ ) [63].

\section{Conclusions}

Virgin olive oil has unique characteristics among all other vegetable oils, and its minor compounds are of paramount importance for their health properties and peculiar sensory notes, in terms of aroma and taste. However, a sole VOO does not exist, as the olive variety, field management, olive fruit maturity degree, harvesting and further processing affect dramatically the final VOO characteristics. At industrial level, the extraction conditions applied can affect the concentration and composition of phenolic and volatile compounds, with possible negative consequence and the formation of offflavours. Olive crushing, malaxation, centrifugation, filtration and storage were reported to be all possible factors affecting VOO flavour.

The factors affecting the presence and amount of positive aroma compounds, as well as the generation of volatile related to the off-flavour should be known to produce a VOO with improved sensory and nutritional characteristics, by the modulation of agronomic and technological factors. These factors include the harvesting, crushing, malaxation, centrifugation, filtration and storage conditions which can all dramatically influence the composition and final flavour of the product. All these major variables have been reviewed in detail in this paper to have a wide vision of the complexity of this product, with particular emphasis on the volatile compounds related to VOO aroma.

\section{References}

1. International Olive Council (IOC). 2015. World olive oil production statistics.

2. EC Regulation No. 2568/91/EEC, 1991. Official Journal of the European Communities L248, pp 1-83.

3. Caporaso N, Savarese M, Paduano A, Guidone G, De Marco E, et al. 2015. Nutritional quality assessment of extra virgin olive oil from the Italian retail market: Do natural antioxidants satisfy EFSA health claims?. J Food Compost Anal 40: 154-162.

4. Angerosa F, Basti C, Vito R. 1999. Virgin olive oil volatile compounds from lipoxygenase pathway and characterization of some Italian cultivars. J Agric Food Chem 47(3): 836-839. doi: 10.1021/jf980911g

5. Benincasa MMP. 2003. Plant Growth Analysis: Basic Knowledge. $2^{\text {nd }}$ ed, Funep, Jaboticabal, pp 41.

6. Boskou D. 2008. Olive Oil: Minor Constituents and Health. CRC Press.

7. Visioli F, Poli A, Gall C. 2002. Antioxidant and other biological activities of phenols from olives and olive oil. Med Res Rev 22(1): 6575. doi: $10.1002 / \operatorname{med} .1028$

8. Piperno A, Toscano M, Uccella NA. 2004. The Cannizzaro-like metabolites of secoiridoid glucosides in some olive cultivars. J Sci Food Agric 84(4): 341-349. doi: 10.1002/jsfa. 1640

9. Conde C, Delrot S, Geros H. 2008. Physiological, biochemical and molecular changes occurring during olive development and ripening. J Plant Physiol 165(15): 1545-1562. doi: 10.1016/j.jplph.2008.04.018

10. Kalua CM, Bedgood DR, Bishop AG, Prenzler PD. 2006. Changes in volatile and phenolic compounds with malaxation time and temperature during virgin olive oil production J Agric Food Chem 54(20): 7641-7651. doi: $10.1021 /$ jf061122z 
11. Angerosa F. 2002. Influence of volatile compounds on virgin olive oil quality evaluated by analytical approaches and sensor panels. Eur J Lipid Sci Technol 104(9-10): 639-660. doi: 10.1002/1438-9312(200210)104:9/10<639::AID-EJLT639>3.0.CO;2-U

12. Kiritsakis AK. 1998. Flavour components of olive oil - A review. J Am Oil Chem Soc 75(6): 673-681. doi: 10.1007/s11746-998-0205-6

13. Olias JM, Perez AG, Rios JJ, Sanz LC. 1993. Aroma of virgin olive oil: biogenesis of the "green" odor notes. J Agric Food Chem 41(12): 23682373. doi: $10.1021 / \mathrm{jf00036a029}$

14. Sanchez J, Harwood JL. 2002. Biosynthesis of triacylglycerols and volatiles in olives. Eur J Lipid Sci Technol 104(9-10): 564-573. doi: 10.1002/1438-9312(200210)104:9/10<564::AID-EJLT564>3.0.CO;2-5

15. Salas JJ, Sanchez J. 1999. The decrease of virgin olive oil flavor produced by high malaxation temperature is due to inactivation of hydroperoxide lyase. J Agric Food Chem 47(3): 809-812. doi: 10.1021/jf981261j

16. Tura D, Failla O, Bassi D, Pedo S, Serraiocco A. 2008. Cultivar influence on virgin olive (Olea europea L.) oil flavor based on aromatic compounds and sensorial profile. Sci Hortic 118(2): 139-148. doi: 10.1016/j.scienta.2008.05.030

17. Montedoro G, Bertuccioli M, Anichini F. 1987. Aroma analysis of virgine olive oil by head space volatiles extraction techniques, In: Flavor of Foods and Beverages, Academic Press, New York, USA, pp 247-281. doi:10.1016/B978-0-12-169060-1.50023-0

18. Angerosa F, Servili M, Selvaggini R, Taticchi A, Esposito S, et al. 2004 Volatile compounds in virgin olive oil: occurrence and their relationship with the quality. $J$ Chromatogr A 1054(1-2): 17-31. doi: 10.1016/j. chroma.2004.07.093

19. Skevin D, Rade D, Strucelj D, Mokrovcak Z, Nederal S, et al. 2003. The influence of variety and harvest time on the bitterness and phenolic compounds of olive oil. Eur J Lipid Sci Technol 105(9): 536-541. doi: 10.1002/ejlt.200300782

20. Dhifi W, Hamrouni I, Ayachi S, Chahed T, Saidani M, et al. 2004. Biochemical characterization of some tunisian olive oils. J Food Lipids 11(4): 287-296. doi: 10.1111/j.1745-4522.2004. 01148.x

21. Baccouri O, Bendini A, Cerretani L, Guerfel M, Baccouri B, et al. 2008. Comparative study on volatile compounds from Tunisian and Sicilian monovarietal virgin olive oils. Food Chem 111(2): 322-328. doi: 10.1016/j.foodchem.2008.03.066

22. Ranalli A, Malfatti A, Cabras P. 2001. Composition and quality of pressed virgin olive oils extracted with new enzyme processing aid. $J$ Food Sci 66(4): 592-603. doi: 10.1111/j.1365-2621. 2001.tb04607.x

23. Salvador MD, Aranda F, Fregapane G. 2001. Influence of fruit ripening on 'Cornicabra'virgin olive oil quality A study of four successive crop seasons. Food Chemistry 73(1): 45-53. doi:10.1016/S03088146(00)00276-4a

24. Haralampidis K, Milioni D, Sanchez J, Baltrusch M, Heinz E, et al. 1998. Temporal and transient expression of stearoyl-ACP carrier protein desaturase gene during olive fruit development. $J \operatorname{Exp}$ Bot 49(327): 1661-1669. doi: 10.1093/jxb/49.327.1661s

25. Lerma-García MJ, Ramis-Ramos G, Herrero-Martínez JM, SimóAlfonso EF. 2008. Classification of vegetable oils according to their botanical origin using sterol profiles established by direct infusion mass spectrometry. Rapid Commun Mass Spectrom 22(7): 973-978. doi: $10.1002 / \mathrm{rcm} .3459$

26. García JM, Seller S, Pérez-Camino MC. 1996. Influence of fruit ripening on olive oil quality. J Agric Food Chem 44(11): 3516-3520. doi: $10.1021 / \mathrm{j} f 950585 \mathrm{u}$

27. Uceda M, Frias L. 1975. Época de recolección. Evolución del contenido graso y de la composición y la calidad del aceite. In: Proceeding II Seminario Oleícola Internacional; Córdoba, Spain.

28. Aparicio R, Morales MT. 1998. Characterization of olive ripeness by green aroma compounds of virgin olive oil. J Agric Food Chem 46(3): 1116-1122. doi: 10.1021/jf970540o

29. Sakouhi F, Absalon C, Sebei K, Fouquet E, Boukhchina S, et al. 2009.
Gas chromatographic-mass spectrometric characterization of triterpene alcohols and monomethyl sterols in developing Olea europaea L. fruits. Food chem 116(1): 345-350. doi: 10.1016/j.foodchem.2009.01.094

30. Rotondi A, Bendini A, Cerretani L, Mari M, Lercker G. 2004. Effect of olive ripening degree on the oxidative stability and organoleptic properties of cv. Nostrana di Brisighella extra virgin olive oil. J Agric Food chem 52(11): 3649-3654. doi: 10.1021/jf049845a

31. Gomez-Rico A, Salvador MD, Greca ML, Fregapane G. 2006. Phenolic and volatile compounds od extra virgin olive oil (Olea europaea $L$. cv. Cornicabra) with regard to fruit ripening and irrigation management. $J$ Agric Food Chem 54(19): 7130-7136. doi: 10.1021/jf060798r

32. Luna G, Aparicio R. 2002. Characterization of monovarietal virgin olive oils. Eur J Lipid Sci Technol 104(9-10): 614-627. doi: 10.1002/14389312(200210)104:9

33. Toker C, Aksoy U, Ertaş H. 2015. The effect of fruit ripening, altitude and harvest year on volatile compounds of virgin olive oil obtained from the Ayvalık variety. Flavour Fragr J. doi: 10.1002/ff.3300

34. Gomez-Caravaca AMC, Cerretani L, Bendini A, Carretero AS, Gutierrez AR, et al. 2008. Effects of fly attack (Bactrocera oleae) on the phenolic profile and selected chemical parameters of olive oil. J Agr Food Chem 56(12): 4577-4583. doi: 10.1021/jf800118t

35. Angerosa F, Di Giacinto L, Solinas M. 1992. Influence of Dacus Oleae infestation on flavor of oils, extracted from attacked olive fruits, by HPLC and HRGC analyses of volatile compounds. Grasas y Aceites 43(3): 134-142. doi: 10.3989/gya. 1992.v43. i3.1165

36. Tamendjari A, Angerosa F, Mettouchi S, Bellal MM. 2009. The effect of fly attack (Bactrocera oleae) on the quality and phenolic content of Chemlal olive oil. Grasas y aceites 60(5): 509-515. doi:10.3989/ gya.032209

37. Patumi M, d'Andria R, Fontanazza G, Morelli G, Giorgio P, et al. 1999. Yield and oil quality of intensively trained trees of three cultivars of olive (Olea europaea L.) under different irrigation regimes. Journal of Horticultural Science and Biotechnology 74(6): 729-737.

38. Ahmed CB, Rouina BB, Sensoy S, Boukhriss M. 2009. Saline water effects on fruit development, quality and phenolic composition of virgin olive oils, cv. Chemlali. J Agric Food Chem 57(7): 2803-2811. doi: 10.1021/jf8034379

39. Ninfali P, Bacchiocca M, Biagiotti E, Esposto S, Servili M, et al. 2008. A 3-year study on quality, nutritional and organoleptic evaluation of organic and conventional extra-virgin olive oils. $\mathrm{J} \mathrm{Am} \mathrm{Oil} \mathrm{Chem} \mathrm{Soc}$ 85(2): 151-158. doi: 10.1007/s11746-007-1171-0

40. Salas JJ, Sanchez J. 1997. Biogenesis of alcohols present in the aroma of virgin olive oil. In: Williams JP, Khan MD, Lem NW (eds) Physiology, Biochemistry and Molecular Biology of Plant Lipids. Springer, The Netherlands, pp 328-330. doi: 10.1007/978-94-017-2662-7_104

41. Gomez-Rico A, Salvador MD, Fregapane G. 2009. Virgin olive oil and olive fruit minor consistuents as affected by irrigation management based on SWP and TDF as compared to $\mathrm{ET}_{\mathrm{c}}$ in medium-density young olive orchards (Olea europaea L. cv. Cornicabra and Morisca). Food Res Int 42(8): 1067-1076. doi: 10.1016/j.foodres.2009.05.003

42. Stefanoudaki E, Williams M, Chartzoulakis K, Harwood J. 2009. Effect of irrigation on quality attributes of olive oil. J Agric Food Chem 57(15): 7048-7055. doi: 10.1021/jf900862w

43. Tognetti R,D'Andria R,Sacchi R,Lavini A,Morelli G,et al.2007.Deficit irrigation affects seasonal changes in leaf physiology and oil quality of Olea europea (cultivars Frantobio and Leccino). Annals of Applied Biology 150(2): 169-186. doi: 10.1111/j.1744-7348.2007.00117.x

44. Romero N, Saavedra J,Tapia F, Sepúlveda B, Aparicio R. 2016. Influence of agroclimatic parameters on phenolic and volatile compounds of Chilean virgin olive oils and characterization based on geographical origin, cultivar and ripening stage.J Sci Food Agric 96(2): 583-592. doi: $10.1002 /$ jsfa. 7127

45. Montedoro G, Servili M, Baldiol M, Miniati E. 1992. Simple and hydrolyzable phenolic compounds in virgin olive oil. 1. Their extraction, separation and quantitative and semiquantitative evaluation by HPLC. 


\section{J Agric Food Chem 40(9): 1571-1576. doi: 10.1021/jf00021a019}

46. Kalua CM, Allen MS, Bedgood DR Jr., Bishop AG, Prenzler PD, et al. 2007. Olive oil volatile compounds, flavour development and quality: A critical review. Food Chem 100(1): 273-286. doi: 10.1016/j. foodchem.2005.09.059

47. Vichi S, Pizzale L, Conte LS, Buxaderas S, Lopez-Tamames E. 2003. Solid-Phase microextraction in the analysis of virgin olive oil volatile fraction: modifications induced by oxidation and suitable markers of oxidative status. J Agric Food Chem 51(22): 6564-6571. doi: 10.1021/ jf030268k

48. Temime SB, Wael T, Bechir B, Leila A, Douja A, et al. 2006. Changes in olive oil quality of chetoui variety according to origin of plantation. Journal of Food Lipids 13: 88-99. doi: 10.1111/j.1745-4522.2006. 00036.x

49. Issaoui M, Jlamini G, Brahmi F, Dabbou S, Hassine KB, et al. 2009. Effect of the growing area conditions on differentiation between Chemlali and Chetoui olive oils. Food Chem 119(1): 220-225. doi:10.1016/j.foodchem.2009.06.012

50. Luna G, Morales MT, Aparicio R. 2006. Characterization of 39 varietal virgin olive oils by their volatile compositions. Food Chem 98(2): 243252. doi:10.1016/j.foodchem.2005.05.069

51. Angerosa F, Di Giacinto L. 1995. Caratteristiche di qualita dellolio vergine in relazione ai metodi di frangitura. Nota Il (Features of virgin oil quality in relation to the pressing methods. The Note). Riv Ital Sostanze Grasse 72: 1-4.

52. Servili M, Montedoro G. 2002. Contribution of phenolic compounds to virgin olive oil quality. Eur J Lipid Sci Technol 104(9-10): 602613. doi: 10.1002/1438-9312(200210)104:9/10<602::AIDEJLT602>3.0.CO;2-X

53. Angerosa F, Mostallino R, Basti C, Vito R. 2001. Influence of malaxation temperature and time on the quality of virgin olive oils. Food Chem 72(1): 19-28. doi: 10.1016/S0308-8146(00)00194-1

54. Servili M, Taticchi A, Esposto S, Urbani S, Selvaggini R, et al. 2008. Influence of the decrease in oxygen during malaxation of olive paste on the composition of volatiles and phenolic compounds in virgin olive oil.
J Agric Food Chem 56(21): 10048-10055. doi: 10.1021/jf800694h

55. Ranalli A, Contento S, Lucera L, Pavone G, Di Giacomo G, et al. 2004. Characterization of carrot root oil arising from supercritical fluid carbon dioxide extraction. J Agric Food Chem 52(15): 4795-4801. doi: 10.1021/jf049713h

56. Aparicio R, Romero M, Khouri N, Rojas LB, Usubillaga A. 2002. Volatile constituents from the leaves of three Coespeletia species from the Venezuelan Andes. Journal of Essential Oil Research 14(1): 37-39. doi: 10.1080/10412905.2002.9699755

57. Di Giovacchino L, Solinas Mz, Miccoli M. 1994. Aspetti qualitativi e quantitativi delle produzioni olearie ottenute dalla lavorazione delle olive con i differenti sistemi di estrazione. Riv Ital Sostanze Grasse 12: 587-594.

58. Sacchi R, Caporaso N, Paduano A, Genovese A. 2015. Industrial-scale filtration affects volatile compounds in extra virgin olive oil cv. Ravece. Eur LLipid Sci Technol 117(12): 2007-2014.doi: 10.1002/ejlt.201400456

59. Savarese M, Caporaso N, De Marco E, Sacchi R. 2013. Extra virgin olive oil overall quality assessment during prolonged storage in PET containers. Proceedings in GV-Global Virtual Conference 1(1): 674679.

60. Caporaso N, Paduano A, Nicoletti G, Sacchi R. 2013. Capsaicinoids, antioxidant activity, and volatile compounds in olive oil flavored with dried chili pepper (Capsicum annuum). Eur J Lipid Sci Technol 115(12): 1434-1442. doi: 10.1002/ejlt.201300158

61. Caporale G, Policastro, S, Monteleone E. 2004. Bitterness enhancement induced by cut grass odorant (cis-3-hexen-1-ol) in a model olive oil. Food Quality and Preference 15(3): 219-227. doi:10.1016/S09503293(03)00061-2

62. Servili M, Esposto S, Fabiani R, Urbani S, Taticchi A, et al. 2009. Phenolic compounds in olive oil: antioxidant, health and organoleptic activities according to their chemical structure. Inflammopharmacology 17(2): 76-84. doi: 10.1007/s10787-008-8014-y

63. Garcia-Gonzales DL, Tena N, Aparicio R. 2007. Characterization of olive paste volatiles to predict the sensory quality of virgin olive oil. Eur J Lipid Sci Technol 109(7): 663-672. doi: 10.1002/ejlt.200700056 